\title{
Analysis of the role of mobility-lifetime products in the performance of amorphous silicon $\boldsymbol{p}-\boldsymbol{i}-\boldsymbol{n}$ solar cells
}

\author{
J. M. Asensi, ${ }^{\text {a) }}$ J. Merten, C. Voz, and J. Andreu \\ Departament de Física Aplicada i Optica, Universitat de Barcelona, \\ Avinguda Diagonal 647, Planta 4, E-08028 Barcelona, Spain
}

(Received 13 October 1998; accepted for publication 30 November 1998)

\begin{abstract}
An analytical model of an amorphous silicon $p-i-n$ solar cell is presented to describe its photovoltaic behavior under short-circuit conditions. It has been developed from the analysis of numerical simulation results. These results reproduce the experimental illumination dependence of short-circuit resistance, which is the reciprocal slope of the $I(V)$ curve at the short-circuit point. The recombination rate profiles show that recombination in the regions of charged defects near the $p-i$ and $i-n$ interfaces should not be overlooked. Based on the interpretation of the numerical solutions, we deduce analytical expressions for the recombination current and short-circuit resistance. These expressions are given as a function of an effective $\mu \tau$ product, which depends on the intensity of illumination. We also study the effect of surface recombination with simple expressions that describe its influence on current loss and short-circuit resistance. (C) 1999 American Institute of
\end{abstract} Physics. [S0021-8979(99)03705-6]

\section{INTRODUCTION}

The collection mechanism in $a$-Si:H-based $p-i-n$ solar cells can be studied theoretically by means of numerical ${ }^{1-3}$ and analytical models ${ }^{4,5}$. Numerical treatments using computer calculation have often been preferred due to the difficulty of solving the fundamental formulas for analysis (Poisson and continuity equations). However, the interpretation of the experimental behavior of the cell from numerical results is often complicated by the large number of parameters involved. Furthermore, many of the material parameters required are experimentally inaccessible or imperfectly known. Analytical models have the drawback of requiring strong assumptions in order to solve the transport equations, but the simplicity of their solutions allows a straightforward link with the experimental results.

There have been fewer fully analytical attempts to describe the collection mechanism in $a-\mathrm{Si}: \mathrm{H} p-i-n$ solar cells than numerical treatments. The main attempt is probably the uniform-field model of Crandall, ${ }^{4}$ whose main assumptions are: constant electric field, negligible diffusion in the $i$ layer, and the use of the Shockley-Read-Hall expression for recombination as derived for a two-state recombination center. These assumptions lead to a very simple expression for the photocurrent as a function of the two carrier drift lengths. Later, Hubin and Shah ${ }^{5}$ proposed a variation of Crandall's model, in which a more realistic description of recombination in $a-\mathrm{Si}: \mathrm{H}$ is introduced. They consider the amphoteric nature of the dangling bond, the main recombination center in $a-\mathrm{Si}: \mathrm{H}$, and use a recombination function based on a single type of three-state recombination center. In this way, they explain some of the differences between Crandall's analytical results and the more realistic models based on numerical simulation: ${ }^{1}$ for example, this treatment shows that it is

\footnotetext{
${ }^{a)}$ Electronic mail: jmasensi@electra.fao.ub.es
}

the carrier with the shorter drift length that will determine collection.

Recently, we used the uniform-field model of Hubin and Shah to interpret the variable illumination measurement of the short-circuit resistance $R_{\mathrm{sc}}$ of $a-\mathrm{Si}: \mathrm{H} p-i-n$ solar cells: ${ }^{6}$ i.e., the reciprocal slope $(\delta V / \delta I)_{V=0}$ of the $I(V)$ curve at the short-circuit point. Over a wide range of illumination levels, $R_{\mathrm{sc}}$ is inversely proportional to the short-circuit current $I_{\mathrm{sc}}$. In this situation, the $R_{\mathrm{sc}}$ value is related to the voltagedependent photocurrent collection and can be calculated by the uniform-field theory. Thus, if $R_{\mathrm{sc}}$ is plotted as a function of $I_{\mathrm{sc}}$, it is possible to extract the value of an effective $\mu \tau$ product which suitably combines the $\mu \tau$ products of electrons and holes in the layer (more recently, other authors ${ }^{7}$ reported a study which is similar but based on the Crandall theory).

Although the method is straightforward and has been satisfactorily applied as a quantifying tool for the state of degradation of $a-\mathrm{Si}: \mathrm{H}$ solar cells and modules, ${ }^{6}$ some experimental results question the validity of the uniform-field model used to interpret variable illumination measurements:

(a) In general, the $\mu \tau_{\text {eff }}$ value deduced from $R_{\text {sc }}$ applying the uniform-field model is significantly lower (by up to 1 order of magnitude) than the one obtained from photoconductivity in intrinsic material.

(b) The $R_{\mathrm{sc}}$ dependence on illumination level is quasilineal: in most samples $R_{\mathrm{sc}} \propto I_{\mathrm{sc}}^{\gamma}$ where $\gamma<1$ is found. In fact, if the value of $\mu \tau_{\text {eff }}$, deduced applying the uniform-field model [see Eq. (10) in Sec. II) is plotted as a function of $I_{\mathrm{sc}}, \mu \tau_{\text {eff }}$ increases as the illumination level increases (see Fig. 1).

In this article, numerical simulation is used to show that these effects could be correlated with the charged defect states which necessarily exist near the $p-i$ and $i-n$ interfaces. For low and intermediate illumination levels, most of the recombination occurs in these regions. When illumination is 


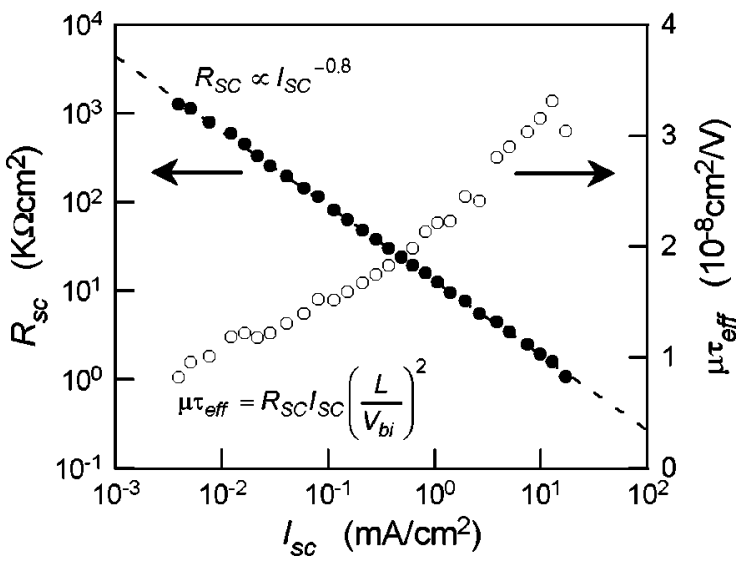

FIG. 1. Variable irradiance measurement of $R_{\mathrm{sc}}$ in $a$-Si:H $p-i-n$ solar cells. The value of $\mu \tau_{\text {eff }}$ deduced from Eq. (10) (uniform-field model) is shown.

increased (for $I_{\mathrm{sc}}>10 \mathrm{~mA} / \mathrm{cm}^{2}$ ), the charged defects are neutralized and the importance of the recombination near the interfaces decreases. Only in this case of high illumination are the uniform-field model assumptions valid. From the numerical results we develop a more detailed analytical description of collection in $p-i-n a-\mathrm{Si}: \mathrm{H}$ solar cells. Our description includes the prominent role of charged defects in the $i$ layer and enables a more general $\mu \tau_{\text {eff }}$ depending on light intensity to be defined.

The paper is organized as follows. In Sec. II, we review the assumptions of the uniform-field model of Shah and $\mathrm{Hu}-$ bin, and show the expression deduced for short-circuit resistance $R_{\mathrm{sc}}$ as a function of the standard effective $\mu \tau$ product. In Sec. III, we describe our numerical model and present the full set of equations used in the computer simulation. Our numerical treatment was simplified for a better comparison with the results of the uniform-field model description. We then simulate a variable illumination measurement of $R_{\mathrm{sc}}$ and show that recombination in the charged regions at interfaces is not negligible. In Sec. IV we present the analytical description of the $p-i-n$ solar cell including the effect of the charged regions. We show that, in thermodynamic equilibrium, the electric field profile, and the widths of the charged regions near the $p-i$ and $i-n$ interfaces can be deduced employing dangling bond statistics. In particular, we show that the use of the "thin solar cell" approach leads to straightforward expressions. We then study the effect of illumination on the electric field, carrier density, and recombination profiles. From this analysis the recombination current and the short-circuit resistance can be given as a function of a new effective $\mu \tau$ product which adequately combines the effect of the different regions on the $i$ layer. Section IV closes with an analysis of the influence of surface recombination.

\section{UNIFORM-FIELD MODEL AND $\mu \tau$ PRODUCT}

Hubin and Shah ${ }^{5}$ solved the problem of bulk collection in a $p-i-n$ solar cell under these three basic assumptions:

(a) constant electric field in the $i$ layer,

(b) negligible diffusion in the $i$ layer, (c) bulk recombination in the $i$ layer is determined by the neutral dangling bonds.

The two first assumptions are indeed applicable to thin $p-i-n$ cells under small or negative voltage bias. These are the same restrictive assumptions as in Crandall's model. To deal with recombination by neutral dangling bonds, they introduce a linear approximation for the recombination function associated with a single type of recombination center that can exist in three charge states: ${ }^{8}$

$$
R_{\mathrm{DB}}=\frac{n}{\tau_{n}^{0}}+\frac{p}{\tau_{p}^{0}},
$$

where $n$ and $p$ are the densities of free carriers (electrons and holes) and $\tau_{n}^{0}$ and $\tau_{p}^{0}$ are the capture times of free electrons and free holes, respectively, by neutral dangling bonds. The capture times are defined by

$$
\tau_{n}^{0}=\left(v_{\mathrm{th}} \sigma_{n}^{0} N_{\mathrm{DB}}\right)^{-1}
$$

and

$$
\tau_{p}^{0}=\left(v_{\mathrm{th}} \sigma_{p}^{0} N_{\mathrm{DB}}\right)^{-1},
$$

where $\sigma_{n}^{0}$ and $\sigma_{p}^{0}$ are the capture cross sections of the free carriers by the neutral dangling bonds, $N_{\mathrm{DB}}$ is the total density of dangling bonds, and $v_{\text {th }}$ is the thermal velocity.

Now, assuming a uniform generation rate $G$ due to weakly absorbed light, the steady-state continuity and transport equations with the appropriate boundary conditions can be solved and the densities of free carriers as a function of the position $x$ in the $i$ layer can be obtained. On introducing $n(x)$ and $p(x)$ into Eq. (1), the total recombination in the $i$ layer can be calculated, and from this the bulk collection $\chi$ (i.e., the fraction of the collected photocurrent divided by the total generation current in the $i$ layer). Hubin and Shah found

$$
\begin{aligned}
\chi= & \frac{1}{L} \frac{l_{n} l_{p}}{l_{n} \exp \left(L / L_{C}\right)-l_{p} \exp \left(-L / L_{C}\right)} \\
& \times\left[\exp \left(\frac{L}{L_{C}}\right)-\exp \left(-\frac{L}{L_{C}}\right)\right],
\end{aligned}
$$

where $L$ is the thickness of the $i$ layer and $L_{C}$ is the collection length:

$$
L_{C}=2 \frac{l_{n} l_{p}}{l_{n}-l_{p}},
$$

where $l_{n}$ and $l_{p}$ are the drift lengths for free electrons and free holes. These lengths depend on the electric field in the $i$ layer $\left(E_{i}\right)$, the band mobilities for free carriers $\left(\mu_{n}\right.$ and $\left.\mu_{p}\right)$, and the capture times of free carriers by neutral dangling bonds $\left(\tau_{n}^{0}\right.$ and $\left.\tau_{p}^{0}\right)$ :

$$
l_{n}=\mu_{n} \tau_{n}^{0}\left|E_{i}\right|
$$

and

$$
l_{p}=\mu_{p} \tau_{p}^{0}\left|E_{i}\right| .
$$

In the case of a thin $p-i-n$ device, the electric field strength is strong enough for the drift lengths to be much larger than the $i$ layer thickness. Then Eq. (3) becomes 


$$
\chi=\frac{L_{C}^{*}}{L_{C}^{*}+L},
$$

where $L_{C}^{*}$ is a redefinition of the collection length as

$$
L_{C}^{*}=2 \frac{l_{n} l_{p}}{l_{n}+l_{p}}=\mu \tau_{\mathrm{eff}}\left|E_{i}\right|,
$$

where $\mu \tau_{\text {eff }}$ is an effective $\mu \tau$ product which suitably combines $\mu \tau$ products of electrons and holes:

$$
\mu \tau_{\mathrm{eff}}=2 \frac{\mu_{n} \tau_{n}^{0} \cdot \mu_{p} \tau_{p}^{0}}{\mu_{n} \tau_{n}^{0}+\mu_{p} \tau_{p}^{0}} .
$$

It can be shown that if $l_{n} \approx l_{p}$ then Eq. (6) is also valid in the more general situation, i.e., when the drift lengths $l_{n}$ and $l_{p}$ are comparable to or shorter than the $i$ layer thickness (see Ref. 5). Note that in this case $L_{C}^{*} \approx l_{n} \approx l_{p}$.

In accordance with Eq. (6) the loss current $I_{\text {rec }}$ in the $i$ layer can be expressed as

$$
I_{\mathrm{rec}}=\frac{L}{L_{C}^{*}} I_{\mathrm{ph}}=\frac{L^{2}}{\mu \tau_{\mathrm{eff}}\left(V_{\mathrm{bi}}-V\right)} I_{\mathrm{ph}},
$$

where $I_{\mathrm{ph}}$ is the generation current in the $i$ layer $\left(I_{\mathrm{ph}}\right.$ $=q G L), V_{\mathrm{bi}}$ is the built-in voltage, and $V$ is the applied voltage.

In the short-circuit region, and neglecting the effect of "parasite" resistance (see Ref. 6), the slope of the $I(V)$ curve is determined by the voltage dependence of $I_{\text {rec }}$. Thus, differentiating Eq. (9) with respect to the applied voltage, the short-circuit resistance can be deduced:

$$
R_{\mathrm{sc}} \approx\left(\frac{d V}{d I_{\mathrm{rec}}}\right)_{V=0}=\mu \tau_{\mathrm{eff}}\left(\frac{V_{\mathrm{bi}}}{L}\right)^{2} I_{\mathrm{sc}}^{-1},
$$

where the generation current $I_{\mathrm{ph}}$ is approximated to the shortcircuit current $I_{\mathrm{sc}}$ (note that we assume $\chi \approx 1$ ). So if we plot $R_{\mathrm{sc}}$ as a function of $I_{\mathrm{sc}}$ it is possible to extract, from the region where $R_{\mathrm{sc}}$ is inversely proportional to $I_{\mathrm{sc}}$, the value of $\mu \tau_{\text {eff }}$.

\section{NUMERICAL SIMULATION}

\section{A. Simplifying assumptions}

All numerical calculations in this article were carried out using the simulation model which was previously developed by our group. ${ }^{3}$ Our computer program uses finite differences and the Newton technique to solve Poisson's equation and continuity equations for the complete diode. The flexibility of the program allows different model assumptions to be analyzed. Our aim here is to study the validity of the hypotheses of the uniform-field model [assumptions (a), (b) and (c), in Sec. II] that lead to Eqs. (9) and (10) for the recombination current and short-circuit resistance, respectively. Therefore, as an excessively detailed description of the diode could complicate the analysis, some simplifying assumptions were incorporated into the numerical treatment:

(a) The transport equations were solved only within the intrinsic layer, and boundary conditions were defined at the doped-layer/intrinsic-layer interfaces $(p-i$ and $i-n)$. This as- sumption reduces significantly the number of physical parameters involved: as will be shown later, the doped-layer influence is completely described by only five $i$ layer boundary condition parameters.

(b) To find the trapped charge density and the recombination rate, only the dangling bonds were examined. This is a good assumption for the middle of the $i$ layer, but is inadequate for the regions near the interfaces $p-i$ and $i-n$, where the Fermi level significantly enters the tail states. However, the tail states mainly affect the trapped charge near the interfaces and are thought to create only a small distortion in the magnitude of the electric field. A similar effect is produced by the fixed space charge at the interfaces in the doped layers. In fact, these two effects can be included as a reduction of the built-in potential $V_{\mathrm{bi}}$, one of the boundary conditions of the problem.

(c) The defect distribution throughout the $i$ layer was assumed uniform and constant. This is the standard model of the density of states in $a-\mathrm{Si}: \mathrm{H}$ (and a normal assumption for all uniform-field models). A further simplification is to assume that the defect states in the gap are discrete.

\section{B. Model equations}

The equations that must be solved numerically are Poisson's equation:

$$
\frac{d E}{d x}=\frac{q}{\varepsilon}[(p-n)+Q(p, n)],
$$

relating the derivative of the electric field $E$ to the local charge (free electrons $n$, free holes $p$, and trapped charge $Q$ ); the current density equations, combining the two driving forces of carrier movement, drift and diffusion, with the total hole $\left(j_{p}\right)$ and electron $\left(j_{n}\right)$ currents:

$$
\begin{aligned}
& j_{p}(x)=q \mu_{p} p(x) E(x)-k T \mu_{p} \frac{d p(x)}{d x}, \\
& j_{n}(x)=q \mu_{n} n(x) E(x)+k T \mu_{n} \frac{d n(x)}{d x},
\end{aligned}
$$

and the two continuity equations:

$$
\begin{aligned}
& \frac{d j_{p}(x)}{d x}=q[G-R(p, n)], \\
& \frac{d j_{n}(x)}{d x}=-q[G-R(p, n)],
\end{aligned}
$$

where $G$ is the generation rate and $R(p, n)$ is the recombination rate.

As stated, we assume that the trapped charge and the recombination are only determined by dangling bonds. Thus, the trapped charge $Q$ is

$$
Q=\left[f^{+}(p, n)-f^{-}(p, n)\right] N_{\mathrm{DB}},
$$

where $N_{\mathrm{DB}}$ is the constant density of dangling bonds in the $i$ layer and $f^{+}$and $f^{-}$are the occupation of the positive and negative dangling-bond states: ${ }^{2}$ 


$$
\begin{aligned}
f^{+}(p, n) & =\frac{T^{+}(p, n)}{1+T^{+}(p, n)+T^{-}(p, n)}, \\
f^{-}(p, n) & =\frac{T^{-}(p, n)}{1+T^{+}(p, n)+T^{-}(p, n)},
\end{aligned}
$$

with

$$
\begin{aligned}
& T^{+}(p, n)=\frac{\sigma_{p}^{0} p+\frac{1}{2} \sigma_{n}^{+} N_{C} e^{-\left(E_{C}-E^{+}\right) / k T}}{\sigma_{n}^{+} n+2 \sigma_{p}^{0} N_{V} e^{-\left(E^{+}-E_{V}\right) / k T}}, \\
& T^{-}(p, n)=\frac{\sigma_{n}^{0} n+\frac{1}{2} \sigma_{p}^{-} N_{V} e^{-\left(E^{-}-E_{V}\right) / k T}}{\sigma_{p}^{-} p+2 \sigma_{n}^{0} N_{C} e^{-\left(E_{C}-E^{-}\right) / k T}},
\end{aligned}
$$

where $\sigma_{n}^{0}$ and $\sigma_{p}^{0}$ are the capture cross sections of electrons and holes by neutral dangling bonds, $\sigma_{n}^{+}$is the capture cross section of electrons by positive dangling bonds, $\sigma_{p}^{-}$is the capture cross section of holes by negative dangling bonds, and $E^{+}$and $E^{-}$are the effective energy levels of the $D^{+} \leftrightarrow D^{0}$ and $D^{-} \leftrightarrow D^{0}$ transitions.

The rate of recombination via dangling bonds is given by

$$
\begin{aligned}
R= & v_{\mathrm{th}}\left(p n-n_{i}^{2}\right)\left(\frac{\sigma_{n}^{+} \sigma_{p}^{0}}{\sigma_{n}^{+} n+2 \sigma_{p}^{0} N_{V} e^{-\left(E^{+}-E_{V}\right) / k T}}\right. \\
& \left.+\frac{\sigma_{p}^{-} \sigma_{n}^{0}}{\sigma_{p}^{-} n+2 \sigma_{n}^{0} N_{C} e^{-\left(E_{C}-E^{-}\right) / k T}}\right) f^{0}(p, n) N_{\mathrm{DB}},
\end{aligned}
$$

where $n_{i}$ is the equilibrium intrinsic concentration and $f^{0}$ is the occupation of the neutral dangling bonds:

$$
f^{0}(p, n)=\frac{1}{1+T^{+}(p, n)+T^{-}(p, n)} .
$$

Finally, this set of coupled differential equations must be solved with the appropriate boundary conditions. As stated above, these conditions are defined at the interfaces $p-i$ and $i$ $n$. The first boundary condition refers to the potential difference across the $i$ layer:

$$
V(L)-V(0)=V_{\mathrm{bi}}-V_{\text {ext }},
$$

where $V_{\mathrm{bi}}$ is the built-in potential, i.e., the difference in the electrostatic potential between the $p$ layer and the $n$ layer in equilibrium, and $V_{\text {ext }}$ is the applied voltage. Note that the full built-in voltage is assumed to be applied over the $i$ layer alone, and that the part of the potential lost in the doped layer space-charge regions is neglected.

The remaining boundary conditions define the current densities at the interfaces by effective surface-recombination velocities $S$ for both holes and electrons. However, some simplification is possible: e.g., for majority carriers we can assume that the interfaces behave as ohmic contacts, and the corresponding $S$ values are very high. Therefore, we can assume a constant majority-carrier concentration that is independent of the current density:
TABLE I. Values of parameters used for numerical calculations.

\begin{tabular}{ll}
\hline \multicolumn{1}{c}{ Principal intrinsic material parameters } \\
\hline Band gap $E_{g}(\mathrm{eV})$ & 1.77 \\
Effective densities of states $N_{C}$ and $N_{V}\left(\mathrm{~cm}^{-3}\right)$ & $4 \times 10^{19}$ \\
Electron mobility $\mu_{n}\left(\mathrm{~cm}^{2} / \mathrm{V} / \mathrm{s}\right)$ & 10 \\
Hole mobility $\mu_{p}\left(\mathrm{~cm}^{2} / \mathrm{V} / \mathrm{s}\right)$ & 4 \\
Dangling bond density $N_{\mathrm{DB}}\left(\mathrm{cm}^{-3}\right)$ & $10^{16}$ \\
Energy level of the $D^{+} \leftrightarrow D^{0}$ transition $E^{+}-E_{V}(\mathrm{eV})$ & 0.735 \\
Effective correlation energy $U_{\mathrm{eff}}(\mathrm{eV})$ & 0.3 \\
Capture cross-section of electrons by $D^{0} \sigma_{n}^{0}\left(\mathrm{~cm}^{2}\right)$ & $5 \times 10^{-16}$ \\
Capture cross-section of electrons by $D^{+} \sigma_{n}^{+}\left(\mathrm{cm}^{2}\right)$ & $2.5 \times 10^{-14}$ \\
Capture cross-section of holes by $D^{0} \sigma_{p}^{0}\left(\mathrm{~cm}^{2}\right)$ & $10^{-16}$ \\
Capture cross-section of holes by $D^{-} \sigma_{p}^{-}\left(\mathrm{cm}^{2}\right)$ & $5 \times 10^{-15}$ \\
Capture times of free carriers by dangling bonds & \\
\hline Capture time of electrons by $D^{0} \tau_{n}^{0}=\left(v_{\mathrm{th}} \sigma_{n}^{0} N_{\mathrm{DB}(}\right)^{-1}(\mathrm{~s})$ & $2 \times 10^{-8}$ \\
Capture time of electrons by $D^{+} \tau_{n}^{+}=\left(v_{\mathrm{th}} \sigma_{n}^{+} N_{\mathrm{DB}}\right)$ & $4 \times 10^{-1}(\mathrm{~s})$ \\
Capture time of holes by $D^{0} \tau_{p}^{0}=\left(v_{\mathrm{th}} \sigma_{p}^{0} N_{\mathrm{DB}}\right)^{-1}(\mathrm{~s})$ & $10^{-7}$ \\
Capture time of holes by $D^{-} \tau_{p}^{-}=\left(v_{\mathrm{th}} \sigma_{p}^{-} N_{\mathrm{DB}}\right)^{-1}(\mathrm{~s})$ & $2 \times 10^{-9}$ \\
\end{tabular}

Doped material parameters

Fermi-level in the $p$-layer $E_{F}-E_{V}(\mathrm{eV})$

Fermi-level in the $n$-layer $E_{C}-E_{F}(\mathrm{eV}) \quad 0.58$

Interface recombination velocity of minority carriers $S_{L} \quad 10$ and $S_{0}(\mathrm{~cm} / \mathrm{s})$

$$
p(0)=p_{\text {eq }}(0)
$$

and

$$
n(L)=n_{\mathrm{eq}}(L),
$$

where $p_{\text {eq }}(0)$ and $n_{\text {eq }}(L)$ are the equilibrium hole and electron densities in the doped layers. For the minority-carrier currents $\left[j_{p}(L)\right.$ and $\left.j_{n}(0)\right]$ we use the more general form:

$$
\begin{aligned}
& j_{p}(L)=q S_{L}\left[p(L)-p_{\mathrm{eq}}(L)\right], \\
& j_{n}(0)=q S_{0}\left[n(0)-n_{\mathrm{eq}}(0)\right],
\end{aligned}
$$

where $p_{\text {eq }}(L)$ and $n_{\text {eq }}(0)$ are the equilibrium minority-carrier densities in the doped layers, and $S_{L}$ and $S_{0}$ are the interface recombination velocities for the minority carriers at the interfaces. Note that the currents given by Eq. (21) are loss currents. The case $S_{L}=S_{0}=0$ is an ideal situation where the interfaces are perfectly blocking contacts for the minority carriers.

\section{Simulation results}

Variable irradiance measurement of $R_{\mathrm{sc}}$ over a range of illumination levels from $10^{-5}$ to $10^{2} \mathrm{~mA} / \mathrm{cm}^{2}$ (for $I_{\mathrm{sc}}$ ) was simulated. It was considered uniform-light illumination. The device simulated was a $0.3-\mu \mathrm{m}$-thick $a$-Si:H solar cell. Model parameters are listed in Table I. These parameters are the typical ones for $a-\mathrm{Si}: \mathrm{H}$ material in the annealed state (e.g., see Ref. 9): with these parameters and using Eq. (8) we obtain a $\mu \tau_{\text {eff }}$ value of $2.7 \times 10^{-7} \mathrm{~cm}^{2} / \mathrm{V}$, and applying Eq. (7) we find $62 \mu \mathrm{m}$ for the collection length $L_{C}^{*}$ (note that this is much longer than the $i$ layer thickness $L$ ).

Figure 2 shows the calculated short-circuit resistance $R_{\mathrm{sc}}$ and the $\mu \tau_{\text {eff }}$, deduced from $R_{\mathrm{sc}}$ by applying Eq. (10) as a function of the short-circuit current $I_{\mathrm{sc}}$. At the lowest illu- 


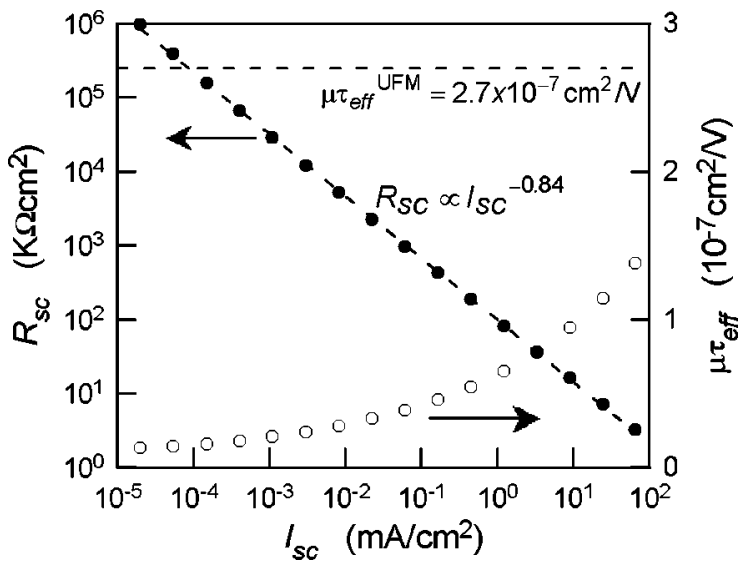

FIG. 2. Simulated short-circuit resistance $R_{\mathrm{sc}}$ as a function of the shortcircuit current $I_{\mathrm{sc}}$. The value of $\mu \tau_{\text {eff }}$ deduced from $R_{\mathrm{sc}}$ by applying Eq. (10) and the theoretical value of $\mu \tau_{\text {eff }}$ deduced from (8) (dashed line) are shown.

mination levels we find a value of $\mu \tau_{\text {eff }}$ approximately one order of magnitude smaller that predicted by the uniformfield theory. On the illumination level increasing, this difference decreases and the $\mu \tau_{\text {eff }}$ is close to the value theoretically predicted. In fact, one can see from Fig. 2 that the $R_{\mathrm{sc}}$ dependence on illumination is quasilineal: we find $R_{\mathrm{sc}} \propto I_{\mathrm{sc}}^{\gamma}$ with $\gamma=0.84$. These results are consistent with the experimental data (see Fig. 1). Consequently, a detailed analysis of our simulation results is expected to reveal aspects of the physics of the device that are not included in the conventional uniform-field model.

Figure 3 shows some calculated profiles (density of trapped charge, electric field, and recombination rate) at short-circuit conditions for two different levels of illumination. The most important differences between simulation and the uniform-field model suppositions appear in the lowillumination regime (solid line in Fig. 3). In this regime, neutrality is only maintained in a small region within the $i$ layer. In the regions near to the doped zones, dangling bonds are charged [Fig. 3(A)], altering the electric field [Fig. 3(B)] and clearly the recombination profile [Fig. $3(\mathrm{C})]$. It can be observed that, in this case of low illumination, most of the recombination occurs close to the interfaces where the defects are in the charged state. So the collection, and probably its dependence on the applied voltage, must be controlled by these regions. When illumination increases, the neutral region increases and the charged regions shrink (dashed line in Fig. 3). The field inside the bulk of the $i$ layer grows and the relative weight of the recombination through charged dangling bonds becomes much lower: note that only in the regime of very high illumination $\left(I_{\mathrm{sc}}>10^{2} \mathrm{~mA} / \mathrm{cm}^{2}\right)$ could hypotheses (a) and (c) of the uniform-field model be considered valid.

Now we shift our attention to hypothesis (b) of the uniform-field model, i.e., that photocarrier transport occurs by field-assisted drift. Figure 4 shows the drift and the diffusion components of the hole current density under shortcircuit conditions for the two cases of illumination. It can be seen that near the $p-i$ interface, where holes are the majority carriers, both drift and diffusion contribute to the photocur-

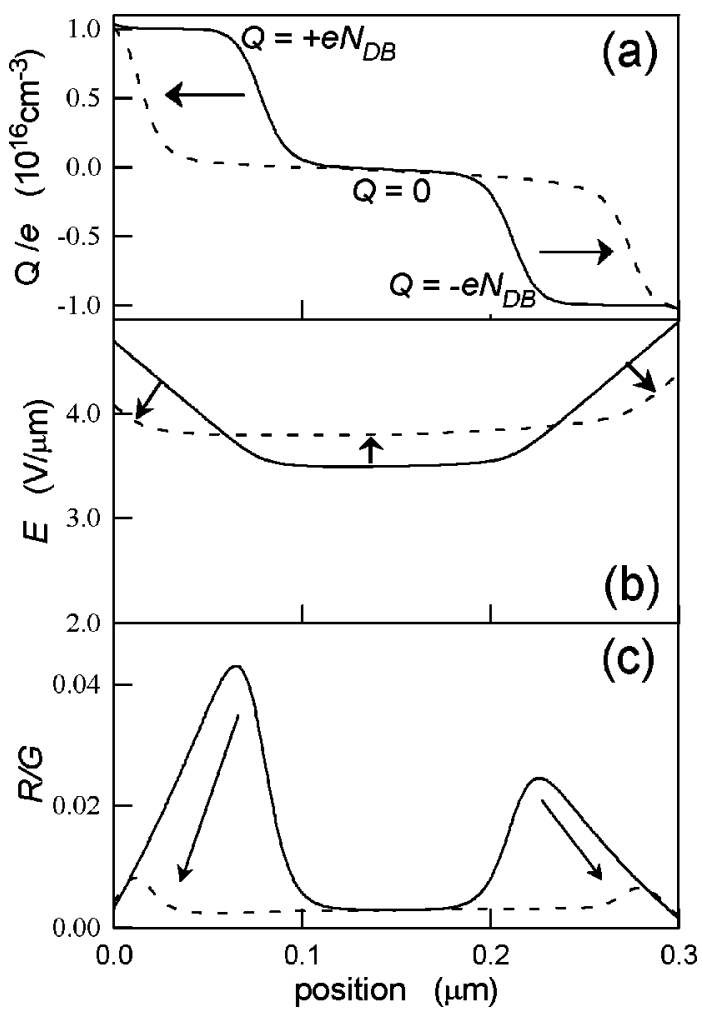

FIG. 3. Simulated profiles of (A) density of trapped charge, (B) electric field, and (C) recombination rate normalized to generation, at short-circuit conditions and for two illumination levels: low (solid line, $I_{\mathrm{sc}}$ $=10^{-3} \mathrm{~mA} / \mathrm{cm}^{2}$ ) and high (dashed line, $I_{\mathrm{sc}}=10^{2} \mathrm{~mA} / \mathrm{cm}^{2}$ ). The arrows indicate evolution with illumination.

rent. Therefore, diffusion cannot be overlooked when solving the hole transport equations in this region. As can also be seen, this effect decreases under very intense illumination and, only in this case, hypothesis (b) of the uniform-field model could be applied in the whole bulk of the $i$ layer. To demonstrate more clearly that diffusion must be included for majority carriers close to the interfaces, in Fig. 5 we compare the profile of photogenerated hole density with the profile deduced from the uniform-field theory which overlooks diffusion [see Eq. (31) in Sec. IV C]. It can be seen that, at low

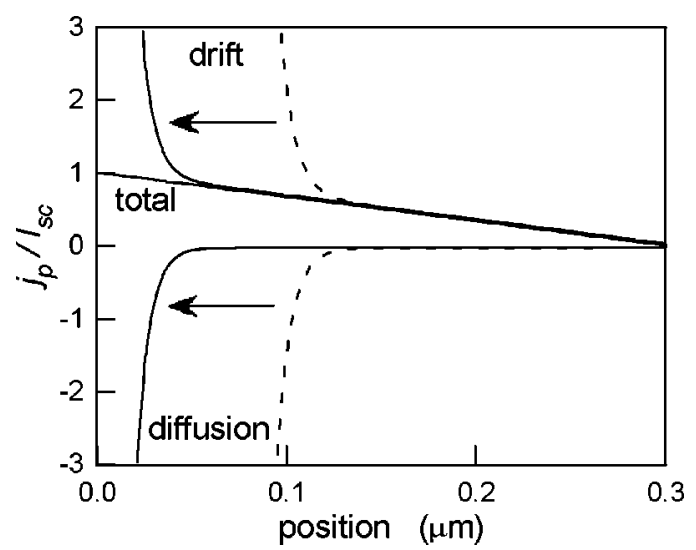

FIG. 4. Simulated profiles of the hole current density normalized to the total short-circuit current in the same conditions as in Fig. 3. The drift and the diffusion components of the current and the effect of the illumination level are shown. 


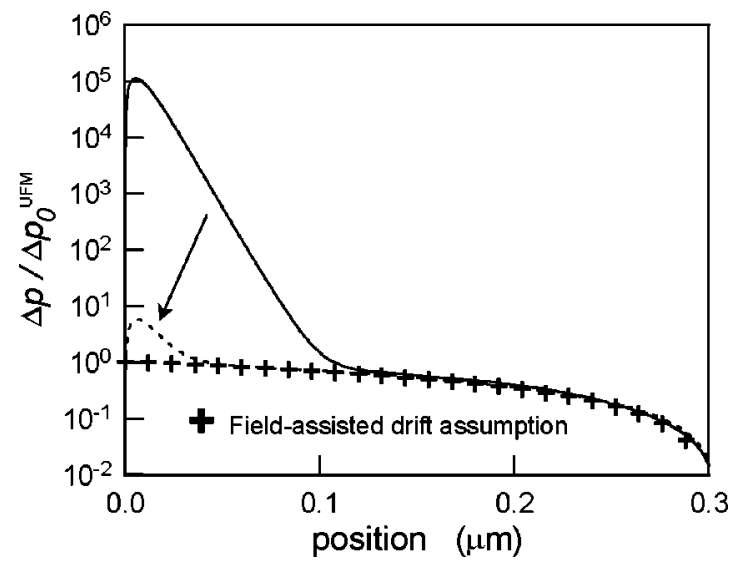

FIG. 5. Photogenerated hole profiles in the same conditions as in Fig. 3. Showing simulated profiles for the two illumination levels, and the theoretical profile obtained by neglecting both diffusion and recombination: $\Delta p(x)=\Delta p_{0}^{\mathrm{UFM}}(L-x)$ with $\Delta p_{0}^{\mathrm{UFM}}=(G \cdot L) /\left(\mu_{p} \cdot V_{\mathrm{bi}}\right)$.

illumination, the simulated photohole density at the $p$ - $i$ interface is significantly different from the theoretical value. As will be discussed later, the majority-carrier densities photogenerated near the interfaces are very sensitive to perturbations in the electric field (which could be due to illumination and/or voltage bias). Although this is not significant in calculating the loss of carrier collection, since recombination in these regions is determined by minority carriers, the complete description of the $p-i-n$ diode must take into account the effect of the majority carriers injected from the $p-i$ and $i$ - $n$ contacts.

In summary, numerical simulation has demonstrated that hypotheses used in the uniform-field model are not fulfilled, especially at low illumination. A correct interpretation of $R_{\mathrm{sc}}$ measurement or, in general, of collection in amorphous $p-i-n$ solar cells must include the state of charge of the defects in the regions close to the doped zones and, probably, the effect of the diffusion current. This is the theme of Sec. IV.

\section{ANALYTICAL DESCRIPTION}

\section{A. Equilibrium}

In thermodynamic equilibrium, i.e., in the dark and without external voltage, the regions near the $p-i$ and $i$ - $n$ interfaces are non-neutral due to the Fermi level shifts in these regions. In Fig. 6, where the band diagram of a $p-i-n$ structure is shown, we can see the different regions in the intrinsic layer. Assuming discrete transition levels for the dangling bonds and using the zero-temperature approximation, three regions within the intrinsic layer can be identified, which vary according to the position of the Fermi level:

(A) Interface region (PI): between $x=0$ and $x=x_{p}$, where $x_{p}$ is the $i$ layer position where $E_{f}$ is on the dangling bond level $E^{+}$. All defects are positively ionized. The potential variation $V_{p}$ across the PI region is determined by the difference between the Fermi level position in the $p$-doped material and the $E^{+}$level of the dangling bond. The electric field strength decreases as a consequence of the defect charge:



FIG. 6. Schematic energy band diagram of $a-\mathrm{Si}: \mathrm{H} p-i-n$ solar cell in equilibrium.

$$
E(x)=E_{0}-q \frac{N_{\mathrm{DB}}}{\varepsilon} x
$$

where the absolute value of the electric field is considered. $E_{0}$ is the field value at the interface $p-i(x=0)$ and $N_{\mathrm{DB}}$ is the defect density.

(B) Bulk region (I): between $x=x_{p}$ and $x=x_{n}$, where $x_{n}$ is the $i$ layer position at which $E_{f}$ is on the dangling bond level $E^{-}$. All defects are neutral and the voltage $V_{i}$ across this region could be determined by the difference between the $E^{+}$and $E^{-}$levels of the dangling bond, i.e., by the correlation energy $U_{\text {eff }}$. The electric field is uniform $[E(x)$ $\left.=E_{i}\right]$.

(C) Interface region (IN): between $x=x_{n}$ and $x=L$. All defects are negatively ionized. The potential variation $V_{n}$ across the IN region is determined by the difference between the Fermi level position in the n-doped material and the $E^{-}$ level of the dangling bond. The electric field strength is

$$
E(x)=E_{L}-q \frac{N_{\mathrm{DB}}}{\varepsilon}(L-x),
$$

where $E_{L}$ is the absolute value of the electric field at the interface $i$ - $n(x=L)$.

Thus, the electric field profile can be expressed in terms of five parameters: $E_{0}, E_{i}, E_{L}, W_{p}$ and $W_{n}$; where $W_{p}$ and $W_{n}$ are the widths of the interface regions $\left(W_{p}=x_{p}\right.$ and $W_{n}=L-x_{n}$ ). These parameters can be obtained as a function of the intrinsic layer thickness $L$ and the potentials $V_{p}, V_{i}$, and $V_{n}$ by solving the following set of equations:

$$
\begin{aligned}
& \left(E_{0}+E_{i}\right) W_{p}=2 V_{p}, \\
& \left(E_{L}+E_{i}\right) W_{n}=2 V_{n}, \\
& E_{i}\left(L-W_{p}-W_{n}\right)=V_{i}, \\
& E_{0}-E_{i}=\frac{q N_{\mathrm{DB}}}{\varepsilon} W_{p}, \\
& E_{L}-E_{i}=\frac{q N_{\mathrm{DB}}}{\varepsilon} W_{n},
\end{aligned}
$$

which is obtained by integrating the electric field profiles across the different regions of the $i$ layer and imposing con- 




FIG. 7. Parameters of the electric field profile in equilibrium as a function of the $i$ layer thickness $L$. Solid lines are theoretical results calculated from Eq. (24). Data points are from numerical simulation. Note that the cell described in Table I is symmetric so that $W_{p}=W_{n}$ and $E_{0}=E_{L}$.

tinuity of the electric field at the limits $x_{p}$ and $x_{n}$. Note that the potential variations $V_{p}, V_{i}$, and $V_{n}$ depend only on the doping level and the energetic position of the dangling bond in the intrinsic material, and the sum of these potentials is the built-in potential $V_{\mathrm{bi}}$ (i.e., the total potential variation across the $i$ layer).

\section{B. "Thin solar cell" approximation}

The set of equations (24) can be solved easily by iterative methods (although it can also be solved analytically, the general solution is not straightforward). Figure 7 shows the dependence of the electric field profile (i.e., the parameters $E_{0}, E_{i}, E_{L}, W_{p}$, and $W_{n}$ ) on the intrinsic layer thickness $L$ for the $p-i-n$ solar cell described in Table I. We also compare the values obtained by solving the set of equations (24) with the values extracted from the numerical results. This plot gives two different kinds of behavior, depending on whether the $i$ layer thickness $L$ is bigger or smaller than a critical thickness value $L^{*}$ related to the widths of the depletion layers in an "infinite thick solar cell:"

$$
L^{*}=W_{p 0}+W_{n 0},
$$

with

$$
W_{p 0}=\sqrt{\frac{2 \varepsilon}{q N_{\mathrm{DB}}} V_{p}}
$$

and

$$
W_{n 0}=\sqrt{\frac{2 \varepsilon}{q N_{\mathrm{DB}}} V_{n}} .
$$

With the cell parameters listed in Table I we obtain $L^{*}$ $\approx 0.5 \mu \mathrm{m}$. At the limit of thick cells (i.e., if $L \gg L^{*}$ ), the widths of the interface regions $W_{p}$ and $W_{n}$ tend to $W_{p 0}$ and $W_{n 0}$, respectively. In this case, the electric field in the $i$ layer departs significantly from uniformity $\left(E_{i} \ll E_{0}\right.$ and $\left.E_{i} \ll E_{L}\right)$. In fact, the set of equations (24), where we use the zerotemperature approximation, leads to $E_{i} \approx V_{i} / L$, while numerical simulation shows that the electric field is much more sensitive to the $i$ layer thickness and $E_{i}$ is virtually zero. This

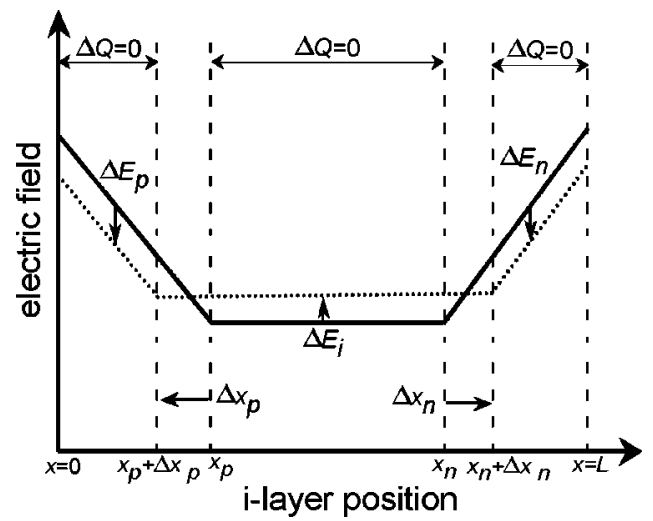

FIG. 8. Perturbation of electric field profile due to illumination.

is a consequence of the trapped charge in the interior of the $i$ layer, near the PI and IN regions, due to the effect of the nonzero temperature.

However, this first study focuses on the most common situation of "thin" solar cells (i.e., when $L<L^{*}$ ). As can be seen in Fig. 7, in this case there is an important and nearly uniform electric field all over the $i$ layer:

$$
E_{i} \approx E_{0} \approx E_{L} \approx \frac{V_{\mathrm{bi}}}{L}
$$

Note that, although in this situation the hypothesis of "uniform field" is a good assumption, the charged regions (PI and IN) in the $i$ layer should not be neglected: the widths $W_{p}$ and $W_{n}$ are an important fraction of the $i$ layer thickness. We find

$$
W_{p} \approx \frac{V_{p}}{V_{\mathrm{bi}}} L
$$

and

$$
W_{n} \approx \frac{V_{n}}{V_{\mathrm{bi}}} L .
$$

\section{Solar cell under uniform illumination}

In general, when the solar cell is under external perturbation (illumination or electrical bias), the profile of charge density changes and, in consequence, the electric field profile also changes. The greatest variation in charge density occurs at the limits $x_{p}$ and $x_{n}$ of the interface regions. In the bulk of these regions the electric charge is mainly due to ionized defects and only a very high illumination level (or applied voltage) can perturb this "fixed" charge. Note that in the bulk of the neutral I region, between $x_{p}$ and $x_{n}$, the effect of the photogenerated space charge could be more important. However we assume, as a first approach, that this effect is not significant. Therefore, we will interpret the perturbation of the charge profile as the variations $\Delta x_{p}$ and $\Delta x_{n}$ for the limits $x_{p}$ and $x_{n}$ of the interface regions (see Fig. 8). As a consequence of this perturbation, the electric field will be modified by the increments $\Delta E_{p}, \Delta E_{i}$ and $\Delta E_{n}$ in the three regions of the cell (note that if $\Delta Q=0$ in the bulk, then $\Delta E$ 
is constant). In the short-circuit condition the following relationships between these increments and the variations $\Delta x_{p}$ and $\Delta x_{n}$ can be found:

$$
\begin{aligned}
& \Delta E_{p}=\Delta E_{i}+\frac{q N_{\mathrm{DB}}}{\varepsilon} \Delta x_{p}, \\
& \Delta E_{n}=\Delta E_{i}-\frac{q N_{\mathrm{DB}}}{\varepsilon} \Delta x_{n}, \\
& \Delta E_{p}\left(W_{p}+\Delta x_{p}\right)+\Delta E_{i}\left(W_{i}-\Delta x_{p}+\Delta x_{n}\right) \\
& +\Delta E_{n}\left(W_{n}-\Delta x_{n}\right)=0,
\end{aligned}
$$

where the first two equations in Eq. (29) are obtained by imposing continuity of the electric field at the new limits of the interface regions, and the last equation $(29 \mathrm{c})$ refers to the short-circuit condition: i.e., the integral of the electric field perturbation across the $i$ layer must be zero.

We need two more equations to calculate the variation in the electric field profile $\left(\Delta E_{p}, \Delta E_{i}, \Delta E_{n}, \Delta x_{p}\right.$, and $\left.\Delta x_{n}\right)$. These can be obtained employing statistics. For example: note that, in thermodynamic equilibrium and using the zerotemperature approach, the limit $x_{p}$ was defined as the position in the $i$ layer at which defects pass from the positive to the neutral state; i.e., $E_{f}\left(x_{p}\right)=E^{+}\left(x_{p}\right)$ (see Fig. 6). Equation (16a) shows that if $T \neq 0$, then at $x_{p}$ the ratio $T^{+}$for positive to neutral defects is $1 / 2$. When the cell is under illumination, this condition will be accomplished at the new limit $x_{p}$ $+\Delta x_{p}$. So, from the more general dangling-bond statistics [see Eqs. (15) and (16)], the following conditions at the new limits of the interface regions can be derived:

$C^{+} \frac{p\left(x_{p}+\Delta x_{p}\right)-p_{\mathrm{eq}}\left(x_{p}\right)}{n\left(x_{p}+\Delta x_{p}\right)-n_{\mathrm{eq}}\left(x_{p}\right)}=C^{-} \frac{n\left(x_{n}+\Delta x_{n}\right)-n_{\mathrm{eq}}\left(x_{n}\right)}{p\left(x_{n}+\Delta x_{n}\right)-p_{\mathrm{eq}}\left(x_{n}\right)}=\frac{1}{2}$,

where $C^{+}$and $C^{-}$are the ratio of capture cross sections for charged to neutral defects: $C^{+}=\sigma_{p}^{0} / \sigma_{n}^{+}$and $C^{-}=\sigma_{n}^{0} / \sigma_{p}^{-}$.

Now, in Eq. (30) we need to know the photogenerated carrier densities in order to solve the coupled set of Eqs. (29) and (30). Assuming that in the neutral region photocarrier transport occurs by field-assisted drift, and neglecting recombination in Eqs. (13a) and (13b) (see Ref. 5), then we arrive at

$$
\Delta p(x) \approx \frac{G(L-x)}{\mu_{p} E(x)}
$$

and

$$
\Delta n(x) \approx \frac{G x}{\mu_{n} E(x)} .
$$

As we discussed at the end of Sec. III, these expressions can be considered valid in the neutral region and valid only for minority carriers in the interface regions (electrons in the PI region and holes in the IN region). For the majority carriers diffusion cannot be ignored and the modified field has to be borne in mind on determining the drift. However, by solving the transport equations in the absence of recombination, simple expressions for the profiles of majority photocarriers are obtained (see Appendix A). So, for the PI region the result is that

$$
\Delta p(x) \approx p_{\text {eq }}(x)\left(e^{-\left(\Delta E_{p} x / V_{T}\right)}-1\right),
$$

where $V_{T}$ is the Boltzmann potential and $p_{\text {eq }}(x)$ is the hole profile in equilibrium which can be calculated from the electric field profile in equilibrium, making $j_{p}(x)=0$ in Eq. (12a):

$$
p_{\text {eq }}(x)=p_{\text {eq }}(0) \exp \left[-\frac{x}{V_{T}}\left(E_{0}-\frac{q N_{\mathrm{DB}}}{2 \varepsilon} x\right)\right] .
$$

Note that the illumination dependence in Eq. (32) is implicit in $\Delta E_{p}$. For photoelectrons in the IN region we arrive at a similar expression, but in the function of $\Delta E_{n}$. Now, using Eqs. (31) and (32) in Eq. (30) and solving the coupled set of equations (29)-(30), we can calculate the perturbation of the electric field profile due to illumination.

In the case of thin solar cells (i.e., when the "thin solar cell" approximation can be applied) a useful simplification is to assume that the electric field increments can be neglected in comparison with the electric field value $E_{i}$ in the $i$ layer. It can be shown that this simplification enables the effect of these increments in Eq. (32) to be removed. Thus, from Eq. (30), we arrive at the following expressions for the thickness variations $\Delta x_{p}$ and $\Delta x_{n}$ of the interface regions:

$$
\begin{aligned}
& C^{+} p_{\text {eq }}\left(x_{p}\right)\left[\exp \left(-\frac{E_{i} \Delta x_{p}}{V_{T}}\right)-1\right]=\frac{G}{\mu_{n} E_{i}}\left(W_{p}+\Delta x_{p}\right), \\
& C^{-} n_{\text {eq }}\left(x_{n}\right)\left[\exp \left(\frac{E_{i} \Delta x_{n}}{V_{T}}\right)-1\right]=\frac{G}{\mu_{p} E_{i}}\left(W_{n}-\Delta x_{n}\right),
\end{aligned}
$$

where $p_{\text {eq }}\left(x_{p}\right)$ and $n_{\text {eq }}\left(x_{n}\right)$ only depend on the position of the electronic defect states:

$$
\begin{aligned}
& p_{\text {eq }}\left(x_{p}\right)=p_{\text {eq }}(0) \exp \left[-\frac{V_{p}}{V_{T}}\right]=N_{V} \exp \left[-\frac{E^{+}-E_{V}}{q V_{T}}\right], \\
& n_{\text {eq }}\left(x_{n}\right)=n_{\text {eq }}(L) \exp \left[-\frac{V_{n}}{V_{T}}\right]=N_{C} \exp \left[-\frac{E_{C}-E^{-}}{q V_{T}}\right] .
\end{aligned}
$$

The equations for $\Delta x_{p}$ and $\Delta x_{n}$ in Eq. (34) are transcendent and must be solved by iterative methods. However, for low perturbation we can assume that $\left|\Delta x_{p}\right| \ll W_{p}$ and $\left|\Delta x_{n}\right|$ $\ll W_{n}$, and then we can obtain analytical solutions for $\Delta x_{p}$ and $\Delta x_{n}$. For example, if $\Delta x_{p}$ is neglected in the right term of Eq. (34a) then we arrive at

$$
\Delta x_{p}=-\frac{V_{T}}{E_{i}} \ln \left(\frac{G W_{p}}{C^{+} p_{\text {eq }}\left(x_{p}\right) \mu_{n} E_{i}}+1\right) .
$$

This result shows that illumination leads to a decrease in the thickness of the interface region.

\section{Voltage dependence of the electric field profile}

In order to evaluate short-circuit resistance, it is necessary to calculate the derivatives of the field profile param- 
eters with respect to the applied voltage. From the electric field profile under short-circuit conditions (i.e., $E_{0}^{*}, E_{i}^{*}$, $E_{L}^{*}, W_{p}^{*}$, and $W_{n}^{*}$, where the superscript * refers to the value under illumination: e.g., $W_{p}^{*}=W_{p}+\Delta x_{p}$ ), and following an analysis similar to the one made in the previous section (see Appendix B), we arrive at

$$
\begin{aligned}
& \left(\frac{\delta E_{i}^{*}}{\delta V}\right)_{V=0}=\left(\frac{\delta E_{0}^{*}}{\delta V}\right)_{V=0}=\left(\frac{\delta E_{L}^{*}}{\delta V}\right)_{V=0}=-\frac{1}{L}, \\
& \left(\frac{\delta W_{p}^{*}}{\delta V}\right)_{V=0}=\frac{W_{p}^{*}}{V_{\mathrm{bi}}} \\
& \left(\frac{\delta W_{n}^{*}}{\delta V}\right)_{V=0}=\frac{W_{n}^{*}}{V_{\mathrm{bi}}}
\end{aligned}
$$

in which "thin solar cell" approximation is included.

\section{E. Recombination and $\mu \tau$ product}

Recombination in the $i$ layer is due to dangling bonds and depends on their charge states in the different regions. Inside the $i$ layer (I region) all defects can be considered as neutral and we can use the linear approximation of the recombination function [Eq. (1)]. In the interface PI region, where defects are positively ionized and electrons are minority carriers, the recombination rate is approximately

$$
R^{p i} \approx \frac{\Delta n}{\tau_{n}^{+}}
$$

with

$$
\tau_{n}^{+}=\left(v_{\mathrm{th}} \sigma_{n}^{+} N_{\mathrm{DB}}\right)^{-1},
$$

where $\tau_{n}^{+}$is the capture time of free electrons by positively ionized dangling bonds and $\sigma_{n}^{+}$is the corresponding capture cross section. The analogous equation for the recombination rate in the IN region is

$$
R^{\text {in }} \approx \frac{\Delta p}{\tau_{p}^{-}}
$$

with

$$
\tau_{p}^{-}=\left(v_{\mathrm{th}} \sigma_{p}^{-} N_{\mathrm{DB}}\right)^{-1},
$$

where $\tau_{p}^{-}$is the capture time of free holes by negatively ionized dangling bonds and $\sigma_{p}^{-}$is the corresponding capture cross section.

To calculate the total recombination $I_{\text {rec }}$ in the $i$ layer, we must take into account the contribution of the different regions:

$$
\begin{aligned}
I_{\mathrm{rec}} & =I_{\mathrm{rec}}^{p i}+I_{\mathrm{rec}}^{i}+I_{\mathrm{rec}}^{i n} \\
& =\int_{0}^{x_{p}^{*}} \frac{\Delta n}{\tau_{n}^{+}} d x+\int_{x_{p}^{*}}^{x_{n}^{*}}\left(\frac{\Delta n}{\tau_{n}^{0}}+\frac{\Delta p}{\tau_{p}^{0}}\right) d x+\int_{x_{n}^{*}}^{L} \frac{\Delta p}{\tau_{p}^{-}} d x,
\end{aligned}
$$

where, as mentioned earlier, $\Delta n$ and $\Delta p$ can be approximated by Eqs. (31) (note that recombination in the interface regions is dependent only on the minority-carrier densities).
Therefore, by solving the integrals in Eq. (40) we can obtain the recombination in the different regions. In the neutral region integration is straightforward and gives

$$
I_{\mathrm{rec}}^{i}=\frac{1}{\mu \tau_{\mathrm{eff}}} \frac{\left(L-W_{p}^{*}-W_{n}^{*}\right)}{E_{i}^{*}} I_{\mathrm{ph}},
$$

where $\mu \tau_{\text {eff }}$ is the same effective $\mu \tau$ product obtained by the standard uniform-field model of Hubin and Shah [i.e., see Eq. (8)). It is important to note that at the limit of very high illumination, $W_{p}^{*}$ and $W_{n}^{*}$ can be ignored in front of $L$, so that the same behavior as in the standard model is found [see Eq. (9)].

At low illumination and, in fact, in a wide range of intermediate illuminations, the widths of the interface regions are important and this means that recombination is determined by the charged defects (with higher capture cross sections than the neutral ones). Integrating Eq. (38) between the limits of the PI region, and using the "thin solar cell" approximation, we arrive at

$$
I_{\mathrm{rec}}^{p i}=\frac{1}{\mu_{n} \tau_{n}^{+}} \frac{W_{p}^{* 2}}{2 L E_{i}^{*}} I_{\mathrm{ph}} .
$$

In the IN region a similar expression can be obtained as a function of the width $W_{n}^{*}$ and the $\mu \tau$ product for negatively ionized dangling bonds $\left(\mu_{p} \tau_{p}^{-}\right)$. Finally, after some manipulation, we arrive at the following expression for the total recombination in the interface regions:

$$
I_{\mathrm{rec}}^{p i+i n}=I_{\mathrm{rec}}^{p i}+I_{\mathrm{rec}}^{i n}=\frac{1}{\mu \tau_{\mathrm{eff}}^{p+i n}} \frac{L}{E_{i}^{*}} I_{\mathrm{ph}},
$$

where a new effective $\mu \tau$ product has been introduced:

$$
\mu \tau_{\mathrm{eff}}^{p i+i n}=2 \frac{\xi^{+} \mu_{n} \tau_{n}^{+} \xi^{-} \mu_{p} \tau_{p}^{-}}{\xi^{+} \mu_{n} \tau_{n}^{+}+\xi^{-} \mu_{p} \tau_{p}^{-}} .
$$

The coefficients $\xi^{+}$and $\xi^{-}$are dimensionless and depend on the ratio of the $i$ layer thickness $L$ to the interface widths:

$$
\xi^{+}=\left(\frac{L}{W_{p}^{*}}\right)^{2}
$$

and

$$
\xi^{-}=\left(\frac{L}{W_{n}^{*}}\right)^{2} .
$$

Note that $\xi^{+}$and $\xi^{+}$depend strongly on illumination. For high illumination levels these coefficients greatly increase and for low illumination levels tend to a constant value which could be evaluated from Eq. (28) (for "thin solar cells").

Now, differentiating Eq. (43) with respect to the applied voltage [including the voltage dependence of $E_{i}^{*}, W_{p}^{*}$ and $W_{n}^{*}$, from Eq. (37)], the short-circuit resistance can be deduced: 


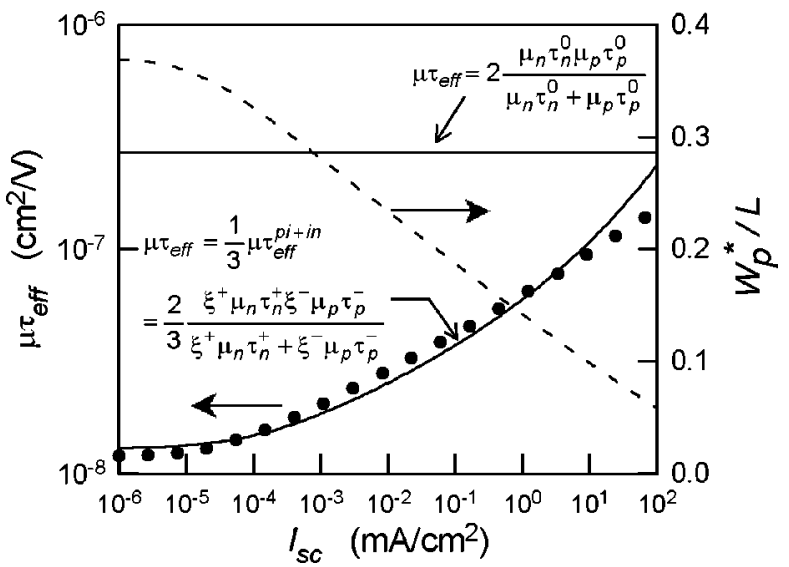

FIG. 9. $\mu \tau_{\text {eff }}$ as a function of $I_{\mathrm{sc}}$. Solid lines are the theoretical values of $\mu \tau_{\text {eff }}$ for the I region and interfaces. Dashed line shows the illumination dependence of the width of the interface PI region deduced from Eq. (34). Data points are the values of $\mu \tau_{\text {eff }}$ deduced from numerical simulation of $R_{\mathrm{sc}} \cdot(L=0.3 \mu \mathrm{m})$.

$$
\begin{aligned}
R_{\mathrm{sc}} & \approx\left(\frac{\delta I_{\mathrm{rec}}^{p i+i n}}{\delta V}\right)_{V=0}^{-1} \\
& =\frac{1}{3} \mu \tau_{\mathrm{eff}}^{p i+i n} E_{i}^{* 2} I_{\mathrm{ph}}^{-1}=\frac{1}{3} \mu \tau_{\mathrm{eff}}^{p i+i n}\left(\frac{V_{\mathrm{bi}}}{L}\right)^{2} I_{\mathrm{ph}}^{-1} .
\end{aligned}
$$

This expression is similar in form to the expression normally deduced from the uniform-field model [Eq. (10)] and, consequently, enables the same method to be used to analyze the variable irradiance measurement of $R_{\mathrm{sc}}$. However, note that the interpretation of the $\mu \tau$ product can be very different.

Figure 9 compares the numerical and analytical $\mu \tau$ product calculations as a function of the short-circuit current $I_{\mathrm{sc}}$ for the $0.3-\mu \mathrm{m}$-thick solar cell with the set of parameters given in Table I. The numerical $\mu \tau$ product is deduced from the simulated short-circuit resistance $R_{\mathrm{sc}}$ as in Fig. 2. The analytical $\mu \tau$ product is separated into its two components: the bulk contribution, i.e., the standard effective $\mu \tau$ product given by Eq. (8) and the interface contribution given by Eq. (44) (to calculate the illumination dependence of the coefficients $\xi^{+}$and $\xi^{-}$in Eq. (44), we employed the most accurate relationships given by Eq. (34)). Note that the total $\mu \tau$ product must be determined by the smaller of the two contributions and, as we can see in Fig. 9, this is precisely the interface contribution.

\section{F. Influence of surface recombination}

Until now we have assumed that the contacts $x=0$ and $x=L$, which define the limits of the $i$ layer, are perfectly blocking for minority carriers: i.e., $S_{L}=0$ and $S_{0}=0$ in Eq. (21). However, minority carriers at the contacts are usually lost by surface recombination, and a current of the opposite sign to the active photocurrent forms. Now we examine the effect of this surface recombination on cells illuminated by uniformly absorbed light under short-circuit conditions. We focus on developing analytical expressions for the current loss at the interfaces and its dependence on voltage bias (i.e., short-circuit resistance).
Under short-circuit conditions, the photocurrent can be obtained by subtracting the different recombination currents from the photogeneration current in the $i$-layer $I_{\mathrm{ph}}$ :

$$
I(V=0)=I_{\mathrm{ph}}-I_{\mathrm{rec}}-I_{\mathrm{rec}}^{S}
$$

where $I_{\text {rec }}$ is the bulk recombination current, which has been discussed already, and $I_{\text {rec }}^{s}$ is the surface recombination, which can be expressed by the sum of the electron current at the $p$ - $i$ interface $(x=0)$ and the hole current at the $n-i$ interface $(x=L)$ :

$$
I_{\mathrm{rec}}^{s}=j_{n}(x=0)+j_{p}(x=L) .
$$

The most simple treatment is to assume that these minority currents are related to the excess of minority carriers at the interfaces according to

$$
\begin{aligned}
& j_{n}(x=0)=q S_{0} \Delta n(0), \\
& j_{p}(x=L)=q S_{L} \Delta p(L),
\end{aligned}
$$

where the interface recombination velocities $S_{0}$ and $S_{L}$ can be considered as constants.

Thus, in order to evaluate $I_{\mathrm{rec}}^{s}$ we need to calculate $\Delta n(0)$ and $\Delta p(L)$, which can be done by solving the transport equations in the regions near the interfaces. For this purpose it is useful to make some simplifying assumptions, the most obvious of which are that bulk recombination is negligible and the electric field is a constant. So, for instance, the electron photocurrent near the $p-i$ interface $(x=0)$ can be given by

$$
j_{n}(x)=-q \mu_{n} \Delta n(x) E_{0}+q V_{T} \mu_{n} \frac{d \Delta n(x)}{d x}
$$

where $E_{0}$ is the absolute value of the electric field. It is important to note that, despite the focus on the transport of minority carriers, the diffusion current is not ignored in Eq. (50). In fact, however strong the electric field $E_{0}$ may be, the assumption of photocarrier transport by field assistance is not correct in a narrow region close to the contact $x=0$ [note that, if $x=0$ in Eq. (50) this assumption leads to $S_{0}$ $=-\mu_{n} E_{0}$, which is incoherent]. In the remaining portion of the PI region the field-assisted transport approach is valid and so this assumption can properly be a boundary condition of our problem: as we move away from the contact, diffusion becomes negligible and the minority-carrier density can be given by Eq. (31).

Thus, using the most general expression, Eq. (50) in the continuity equation for photoelectrons, we arrive at the following differential equation:

$$
\frac{d^{2} \Delta n}{d x^{2}}-\frac{E_{0}}{V_{T}} \frac{d \Delta n}{d x}=-\frac{G}{\mu_{n} V_{T}} .
$$

This equation can be readily integrated, using the boundary condition that $\Delta n(x)=G x / \mu_{n} E_{0}$ at ' $x \rightarrow \infty$,' to give

$$
\Delta n(x)=\Delta n(0)+\frac{G x}{\mu_{n} E_{0}} .
$$


This expression for $\Delta n(x)$ can be used in Eq. (50) to determine the electron photocurrent. Then, we can evaluate the electron photocurrent at $x=0$ and, eliminating $\Delta n(0)$ by means of Eq. (49a), we arrive at

$$
j_{n}(0)=\frac{V_{T}}{E_{0}} \frac{S_{0}}{\left(S_{0}+\mu_{n} E_{0}\right)} G .
$$

For the hole photocurrent at $x=L$ we can find a similar equation but expressed in terms of $S_{L}, \mu_{p}$, and the absolute value $E_{L}$ of the electric field near $x=L$. Now, differentiating Eq. (53) with respect to the applied voltage, we can deduce the contribution of the surface recombination at $x=0$ to the short-circuit resistance. There are two important limiting situations (we include the "thin solar cell" approximation, i.e., $\left.E_{0} \approx E_{i} \approx V_{\mathrm{bi}} / L\right)$ :

(A) Weak surface recombination $\left(S_{0} \ll \mu_{n} E_{i}\right)$ :

$$
\begin{aligned}
& I_{\mathrm{rec}}^{s}=\frac{V_{T}}{V_{\mathrm{bi}}^{2}} \frac{S_{0}}{\mu_{n}} L I_{\mathrm{ph}}, \\
& R_{\mathrm{sc}}=\frac{V_{\mathrm{bi}}^{3}}{2 V_{T}} \frac{\mu_{n}}{S_{0}} L^{-1} I_{\mathrm{ph}}^{-1} .
\end{aligned}
$$

(B) Strong surface recombination $\left(S_{0} \gg \mu_{n} E_{i}\right)$ :

$$
\begin{gathered}
I_{\mathrm{rec}}^{s}=\frac{V_{T}}{V_{\mathrm{bi}}} I_{\mathrm{ph}}, \\
R_{\mathrm{sc}}=\frac{V_{\mathrm{bi}}^{2}}{V_{T}} I_{\mathrm{ph}}^{-1} .
\end{gathered}
$$

A significant aspect of these results is the dependence of $R_{\mathrm{sc}}$ on the $i$ layer thickness $L$ : for weak surface recombination, $R_{\mathrm{sc}}$ is proportional to $L^{-1}$ and, for strong surface recombination, $R_{\mathrm{sc}}$ is independent of $L$. This behavior is different from what is found in the case of bulk recombination, in which $R_{\text {sc }}$, derived from the voltage dependence of recombination in both neutral and interface regions, is proportional to $L^{-2}$.

In order to examine the effect of the surface recombination, and to check the validity of Eqs. (54) and (55), Fig. 10 shows plots of the numerical and analytical $R_{\mathrm{sc}}$ calculations as a function of $S_{0}$ for solar cells with different $i$-layer thickness (the remaining cell parameters are given in Table I). We consider uniform illumination with $I_{\mathrm{ph}}=10^{-3} \mathrm{~mA} / \mathrm{cm}^{2}$. Figure 10 shows that $R_{\mathrm{sc}}$ is only determined by surface recombination in the case of very thin solar cells $(L<0.3 \mu \mathrm{m})$, and at sufficiently high $S_{0}\left(>10^{4} \mathrm{~cm} / \mathrm{s}\right)$.

\section{SUMMARY}

Our numerical simulation results reproduce the experimental data of the illumination dependence of the shortcircuit resistance $R_{\mathrm{sc}}$ in $a-\mathrm{Si}: \mathrm{H} p-i-n$ solar cells. These results suggest that recombination in the charged regions of the $i$ layer should not be overlooked. We then developed a new analytical model to describe collection in $p-i-n$ structures under short-circuit conditions and uniform illumination. The recombination current and the short-circuit resistance can be given as a function of a $\mu \tau$ product which adequately combines two effective $\mu \tau$ products for the different regions in

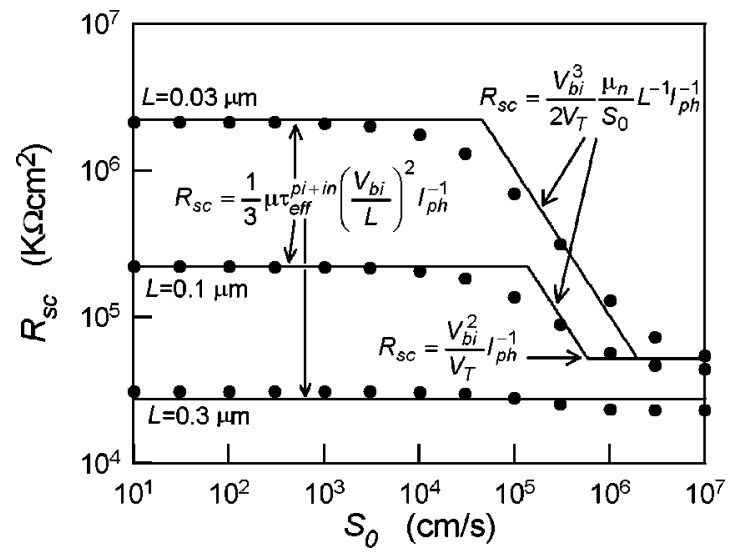

FIG. 10. Short-circuit resistance as a function of the surface recombination rate $S_{0}$ for solar cells with different $i$ layer thickness. It is considered that $I_{\mathrm{ph}}=10^{-3} \mathrm{~mA} / \mathrm{cm}^{2}$. Solid lines are the theoretical values of $R_{\mathrm{sc}}$ (the main contribution is plotted) and data points are the values of $R_{\mathrm{sc}}$ obtained from numerical simulation. (We assume that the contact $x=L$ is perfectly blocking.)

the $i$ layer: (1) for the neutral region in the bulk of the $i$ layer we find the same effective $\mu \tau$ product as is obtained with the standard uniform-field model, (2) for the charged regions at the interfaces we find a new effective $\mu \tau$ product which is light-dependent. We show that recombination and $R_{\mathrm{sc}}$ are determined by this latter $\mu \tau$ product in a wide range of illumination.

We also examined the effect of surface recombination. We demonstrated that, under uniform illumination and shortcircuit conditions, surface recombination could not be negligible in very thin solar cells at sufficiently high surface recombination rates. It could be evaluated by a check on the effect of the $i$-layer thickness on $R_{\mathrm{sc}}$.

We have also shown that, in the analysis of $p-i-n$ solar cells, it is necessary to take into consideration both the diffusion process for majority carriers at interfaces and the effect of the variation in the electric field. We obtained elementary expressions that can be used in analyzing the general behavior of $p-i-n$ solar cells.

\section{ACKNOWLEDGMENTS}

The authors thank Professor A. V. Shah for valuable discussions. This study was supported by the CRYSTAL program of the EC (Grant No. JOR3-CT97-0126).

\section{APPENDIX A: MAJORITY-CARRIER PROFILES IN SHORT-CIRCUIT CONDITIONS}

In the interface regions near the doped layers, the majority-carrier densities and the gradients are important: carrier diffusion from the doped regions cannot be ignored in determining the transport and. moreover, the electric field variation $\Delta E$ caused by illumination can also contribute to the photocurrent. Thus, in the PI region the hole photocurrent, expressed in terms of increments, should be given by 


$$
\begin{aligned}
j_{p}(x) / q= & -\mu_{p} p_{\mathrm{eq}}(x) \Delta E_{p}-\mu_{p}\left[E_{\mathrm{eq}}(x)+\Delta E_{p}\right] \Delta p(x) \\
& -\mu_{p} V_{T} \frac{d \Delta p}{d x}
\end{aligned}
$$

where $p_{\text {eq }}(x)$ is the hole density profile in equilibrium [given by Eq. (33)], $E_{\mathrm{eq}}(x)$ is the electric field profile in equilibrium [note that it is positive, see Eq. (22)], and $\Delta E_{p}$ is the electric field variation in the PI region due to illumination. As has been discussed, the photogenerated space charge in the bulk of the PI region is negligible, and so it can be assumed that $\Delta E_{p}$ is a constant. Thus, introducing Eq. (A1) into the continuity equation, and ignoring recombination, we find the following differential equation for the hole density increment in the PI region:

$$
\begin{aligned}
\frac{d^{2} \Delta p}{d x^{2}} & +\frac{1}{V_{T}}\left[E_{\mathrm{eq}}(x)+\Delta E_{p}\right] \frac{d \Delta p}{d x}-\frac{1}{V_{T}} \frac{q N_{\mathrm{DB}}}{\varepsilon} \Delta p \\
= & -\frac{G}{V_{T} \mu_{p}}-\frac{\Delta E_{p}}{V_{T}} \frac{d p_{\mathrm{eq}}}{d x} .
\end{aligned}
$$

This equation can be solved for $\Delta p$ and gives a relatively complicated expression which is expressed in terms of the error function $\operatorname{Erf}(y)$. It can be demonstrated that this function is well approximated by $1-\mathrm{e}^{-y^{2}} / \sqrt{\pi} y$ and, using the boundary condition that $\Delta p(x=0)=0$ (i.e., assuming ohmic contact), after some manipulation we arrive at

$$
\begin{aligned}
\Delta p(x)= & p_{\mathrm{eq}}(x)\left[(1-C) \exp \left(-\frac{\Delta E_{p} x}{V_{T}}\right)-1\right] \\
& +\frac{\mu_{p} p_{\mathrm{eq}}(0)\left(E_{0}+\Delta E_{p}\right) C-G x}{\mu_{p}\left[E_{\mathrm{eq}}(x)+\Delta E_{p}\right]},
\end{aligned}
$$

where $C$ is the constant of integration that we should obtain by imposing a new condition. To this effect, from Eq. (A3) we can calculate the hole photocurrent in the PI region. It can be demonstrated that only the drift component of the second term on the right-hand side of Eq. (A3) significantly contributes to the photocurrent (the first term gives a diffusion component that is compensated by drift). The result is that

$$
j_{p}(x) / q \approx G x-\mu_{p} p_{\mathrm{eq}}(0)\left(E_{0}+\Delta E_{p}\right) C .
$$

On the other hand, from the continuity equation for holes, neglecting recombination and imposing $j_{p}(L)=0$, we find

$$
j_{p}(x) / q=-G(x-L),
$$

then, equating Eqs. (A4) and (A5) we can determine the value of $C$ :

$$
C=\frac{G L}{\mu_{p} p_{\mathrm{eq}}(0)\left(E_{0}+\Delta E_{p}\right)} .
$$

For a typical solar cell (defined by the set of parameters given in Table I) under high illumination $\left(I_{\mathrm{ph}}=10 \mathrm{~mA} / \mathrm{cm}^{2}\right)$ we find $C \approx 10^{-4}$, so that $C \ll 1$, and so this constant can be safely ignored in the first term of Eq. (A3). Finally, substitution of Eq. (A6) into Eq. (A3) yields

$$
\begin{aligned}
\Delta p(x)= & p_{\mathrm{eq}}(x)\left[\exp \left(-\frac{\Delta E_{p} x}{V_{T}}\right)-1\right] \\
& +\frac{G}{\mu_{p}\left(E_{\mathrm{eq}}(x)+\Delta E_{p}\right)}(L-x)
\end{aligned}
$$

Note that the second term in Eq. (A7) is the photogenerated hole distribution that we obtain making the field-assisted drift assumption [see Eq. (31)] and, as we have seen from Fig. 5, this is only a very small fraction of the total. We thus obtain the relationship given by Eq. (32) for the photogenerated hole profile in the PI region.

\section{APPENDIX B: EFFECT OF APPLIED VOLTAGE (DERIVATIVES)}

We examined a $p-i-n$ solar cell in short-circuit conditions under weakly absorbed light. Illumination alters the electric field profile by the increments $\Delta x_{p}, \Delta x_{n}, \Delta E_{p}, \Delta E_{i}$, and $\Delta E_{n}$, so that the analytical expressions for the profiles of electric field and carrier densities are:

(A) PI region $\left(0<x<W_{p}^{*}\right)$ :

$$
\begin{aligned}
& E^{*}(x)=E_{0}^{*}-\frac{q N_{\mathrm{DB}}}{\varepsilon} x, \\
& p^{*}(x) \approx p_{\mathrm{eq}}(0) \exp \left[-\frac{x}{V_{T}}\left(E_{0}^{*}-\frac{q N_{\mathrm{DB}}}{2 \varepsilon} x\right)\right], \\
& n^{*}(x) \approx \frac{G x}{\mu_{n} E^{*}(x)} .
\end{aligned}
$$

(B) I region $\left(W_{p}^{*}<x<W_{n}^{*}\right)$ :

$E^{*}(x)=E_{i}^{*}$,

$p^{*}(x) \approx \frac{G(L-x)}{\mu_{p} E_{i}^{*}}$,

$n^{*}(x) \approx \frac{G x}{\mu_{n} E_{i}^{*}}$.

(C) IN region $\left(W_{n}^{*}<x<L\right)$ :

$$
\begin{aligned}
& E^{*}(x)=E_{L}^{*}-\frac{q N_{\mathrm{DB}}}{\varepsilon}(L-x), \\
& p^{*}(x) \approx \frac{G(L-x)}{\mu_{p} E^{*}(x)}, \\
& n^{*}(x) \approx n_{\mathrm{eq}}(L) \exp \left[-\frac{(L-x)}{V_{T}}\left(E_{L}^{*}-\frac{q N_{\mathrm{DB}}}{2 \varepsilon}(L-x)\right)\right] .
\end{aligned}
$$

The superscript* in Eqs. (B1)-(B3) refers to the value under illumination. In this situation, if a small external voltage $V$ is applied, then the electric profile will change. The widths $W_{p}^{*}$ and $W_{n}^{*}$ will be modified by the new increments $\Delta x_{p}^{v}$ and $\Delta x_{n}^{v}$, respectively, and, assuming that the variation of the space charge in the bulk of the different regions is negligible, the electric field will be modified by the constants $\Delta E_{p}^{v}$, 
$\Delta E_{i}^{v}$, and $\Delta E_{n}^{v}$. Using an argument similar to the one in Sec. III $\mathrm{C}$, we find the following relationship among these increments:

$$
\begin{aligned}
& \Delta E_{p}^{v}=\Delta E_{i}^{v}+\frac{q N_{\mathrm{DB}}}{\varepsilon} \Delta x_{p}^{v}, \\
& \Delta E_{n}^{v}=\Delta E_{i}^{v}-\frac{q N_{\mathrm{DB}}}{\varepsilon} \Delta x_{n}^{v}, \\
& \Delta E_{p}^{v} W_{p}^{*}+\Delta E_{i}^{v} W_{i}^{*}+\Delta E_{n}^{v} W_{n}^{*}=-V ;
\end{aligned}
$$

where, in the last equation (B4c), we assume that the applied voltage is sufficiently small for $\left|\Delta x_{p}^{v}\right| \ll W_{p}^{*}$ and $\left|\Delta x_{n}^{v}\right|$ $\ll W_{n}^{*}$. The two remaining equations can be obtained, as in Sec. III C, by imposing $T^{+}\left(x_{p}^{*}+\Delta x_{p}^{v}\right)=1 / 2$ and $T^{-}\left(x_{n}^{*}\right.$ $\left.+\Delta x_{n}^{v}\right)=1 / 2$ [i.e., Eq. (30)].

On the other hand, if low applied voltage is assumed, it can be demonstrated that the most significant perturbation of carrier distribution occurs for majority carriers in the interface regions. To obtain the hole profile in the PI region, we can reach a differential equation similar to Eq. (A2) but for the hole density increment due to the electrical bias. Thus, solving the differential equation, we find that the total hole density in the PI region is well approximated by

$$
p(x) \approx p^{*}(x) \exp \left[-\frac{\Delta E_{p}^{v}}{V_{T}} x\right]
$$

where $p^{*}(x)$ is the hole distribution in the PI region for the cell in short-circuit conditions under illumination. Now, introducing Eq. (B5) in the condition $T^{+}\left(x_{p}^{*}+\Delta x_{p}^{v}\right)=1 / 2$ we arrive at

$$
\begin{aligned}
p^{*}\left(x_{p}^{*}\right) \exp \left[-\frac{1}{V_{T}}\left(E_{i}^{*} \Delta x_{p}^{v}+x_{p}^{*} \Delta E_{p}^{v}\right)\right] \\
\approx \frac{G W_{p}^{*}}{\mu_{n} E_{i}^{*} C^{+}}+p_{\text {eq }}\left(x_{p}\right),
\end{aligned}
$$

where the second term could be considered a constant. So, differentiating Eq. (B6) with respect to $V$ we obtain

$$
\frac{d \Delta x_{p}^{v}}{d V}=-\frac{W_{p}^{*}}{E_{i}^{*}} \frac{d \Delta E_{p}^{v}}{d V}
$$

From this equation and differentiating Eq. (B4a), we arrive at the following relationship between the derivatives of $E_{p}^{*}$ and $E_{i}^{*}$ :

$$
\left(\frac{\delta E_{p}^{*}}{\delta V}\right)_{V=0}=\frac{E_{i}^{*}}{E_{0}^{*}}\left(\frac{\delta E_{i}^{*}}{\delta V}\right)_{V=0} .
$$

Using similar reasoning in the IN region, we could arrive at

$$
\left(\frac{\delta E_{n}^{*}}{\delta V}\right)_{V=0}=\frac{E_{i}^{*}}{E_{L}^{*}}\left(\frac{\delta E_{i}^{*}}{\delta V}\right)_{V=0} .
$$

It now remains to calculate the derivative of $E_{i}^{*}$ with respect to $V$. This can be done by differentiating Eq. (B4c) and using Eqs. (B8) and (B9). We find

$$
\left(\frac{\delta E_{i}^{*}}{\delta V}\right)_{V=0}=-\left(\frac{E_{i}^{*}}{E_{0}^{*}} W_{p}^{*}+W_{i}^{*}+\frac{E_{i}^{*}}{E_{L}^{*}} W_{n}^{*}\right)^{-1} .
$$

For "thin" solar cells, it can be shown that this last derivative reduces to $-1 / L$. Also, for high illumination levels, when the neutral I region fills the $i$ layer, the derivative of $E_{i}^{*}$ tends to $-1 / L$.

Other useful relationships are the derivatives of $W_{p}^{*}$ and $W_{n}^{*}$ with respect to $V$. These can be most easily expressed as a function of the derivative of $E_{i}^{*}$

$$
\begin{aligned}
& \left(\frac{\delta W_{p}^{*}}{\delta V}\right)_{V=0}=-\frac{W_{p}^{*}}{E_{0}^{*}}\left(\frac{\delta E_{i}^{*}}{\delta V}\right)_{V=0}, \\
& \left(\frac{\delta W_{n}^{*}}{\delta V}\right)_{V=0}=-\frac{W_{n}^{*}}{E_{L}^{*}}\left(\frac{\delta E_{i}^{*}}{\delta V}\right)_{V=0} .
\end{aligned}
$$

${ }^{1}$ M. Hack and M. Shur, J. Appl. Phys. 58, 997 (1985).

${ }^{2}$ J. L. Gray, IEEE Trans. Electron Devices ED-36, 906 (1989).

${ }^{3}$ J. M. Asensi, J. Andreu, J. Puigdollers, J. Bertomeu, and J. C. Delgado, Mater. Res. Soc. Symp. Proc. 297, 315 (1993).

${ }^{4}$ R. S. Crandall, J. Appl. Phys. 54, 7176 (1983).

${ }^{5}$ J. Hubin and A. V. Shah, Philos. Mag. B 72, 589 (1995).

${ }^{6}$ J. Merten, J. M. Asensi, C. Voz, A. V. Shah, R. Platz, and J. Andreu, IEEE Trans. Electron Devices ED-45, 423 (1998).

${ }^{7}$ S. S. Hegedus, Prog. Photovolt. Res. Appl. 5, 151 (1997).

${ }^{8}$ J. Hubin, A. V. Shah, and E. Auvain, Philos. Mag. Lett. 66, 115 (1992).

${ }^{9}$ A. V. Shah, J. Hubin, E. Sauvain, P. Pipoz, N. Beck, and N. Wyrsch, J. Non-Cryst. Solids 164-166, 485 (1993). 